RESEARCH PAPER

\author{
Available Online at www.ijarcs.info
}

\title{
BIG BANG- BIG CRUNCH BASED SATELLITE IMAGE CLASSIFICATION
}

\author{
Sunita Sharma \\ Research Scholar \\ Mewar University \\ Rajasthan, India
}

\author{
V.K. panchal \\ Founder President \\ Computational Intelligence Research Group \\ New Delhi, India
}

\begin{abstract}
Nature has always been a major source of inspiration to researchers. Nowadays researchers are working on the algorithms that are inspired by the nature. A number of algorithms are proposed which are based on inspiration from nature like Bio Geography based Optimization (BBO), Particle Swarm Optimization (PSO) etc. Recently a new nature inspired algorithm - Big Bang- Big Crunch (BBBC) has been proposed that relies on one of the theories of the evolution of the universe. In the Big Bang phase, particles are randomly spread into universe and in Big Crunch phase, randomly distributed particles are converged to a single point. Satellite image classification is an important task because it is the only way we can know about the land cover map of the inaccessible areas. Although a number of algorithms are proposed for satellite image classification, but there is always a search for alternative strategies which could be best suited for a particular land cover feature extraction task in hand. This paper is focused on classification of satellite image of a particular land cover using the theory of Big Bang- Big Crunch. The original BBBC optimization algorithm does not have the inbuilt property of classification which is required during image classification. Hence original algorithm has been adapted to classify the satellite image of a particular land cover. The results indicate that highly accurate land cover features can be extracted effectively when the proposed adaptations are used.
\end{abstract}

Keywords: Big-Bang Big Crunch, Bio Geography Based Optimization (BBO), Particle Swarm Optimization (PSO), satellite image classification

\section{INTRODUCTION}

A new nature inspired algorithm for optimization called Big Bang-Big Crunch (BBBC) optimization algorithm has been introduced recently by Osman K. Erol et. al. [1]. The algorithm is based upon the famous Big Bang- Big Crunch theory of evaluation of universe. The algorithm has been successfully applied to solve optimization problems in different areas like solving Optimal Power Flow Problems[6], Economic Dispatch with Valve-Point Effect[7], fuzzy rulebased generations from Numerical Data[8], Chaotic based BBBC for solving global optimization problems [9], multi criteria group decision making [10], damage detection [11]. For solving global optimization problems the BBBC algorithm has been shown to outperform the enhanced classical Genetic Algorithm for many Benchmark test functions [1]. Big Bang-Big Crunch algorithm is basically an optimization technique which is used to find optimized solution of a problem, but satellite image classification is a clustering problem that requires each pixel in the image to be classified in some class, hence adaptation in the original algorithm is undertaken and a new BBBC based satellite image classifier is developed.

\section{A. Big Bang Crunch Theory}

Big Bang- Big Crunch theory [15] [16] [17] is one of the famous and highly accepted theories of evaluation of universe. According to the Big Bang - Big Crunch theory, the universe began about twelve to fifteen billion years ago in a violent explosion. During the Big Bang phase, the most basic forces in nature were formed which were gravity and strong nuclear forces followed by the week nuclear and electromagnetic forces and particles like electrons, protons, neutrons, quarks etc were also formed. After the Big Bang Phase the nuclei of simple elements like hydrogen and helium were formed by nucleo-synthesis of protons and neutrons and matter came into existence by the nuclei bounding with the free electrons, and then galaxies and stars were formed by condensing of gases by virtue of Gravity, this phase is known as Big Crunch Phase. So in the Big Bang Phase energy explosion produces randomness and disorder due to which particles are spread randomly whereas in Big Crunch phase the randomly distributed particles are drawn into an order and converged to an optimal point by gravitational attractions, nuclear forces etc. as shown in the figure 1.1 and 1.2.

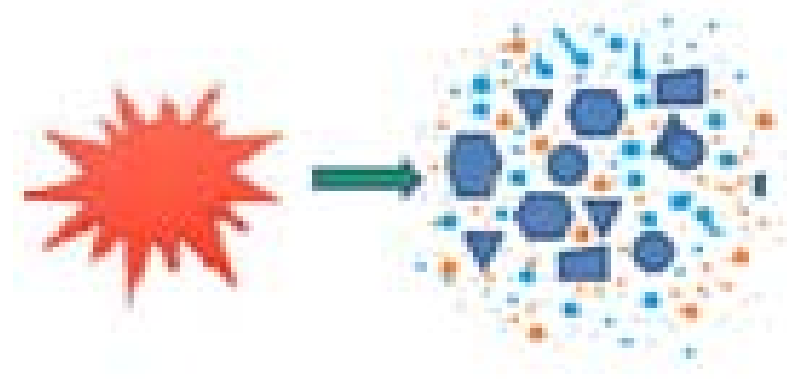

Blast in Nature Random Spread of Particles

Figure 1.1: Big Bang Phase 

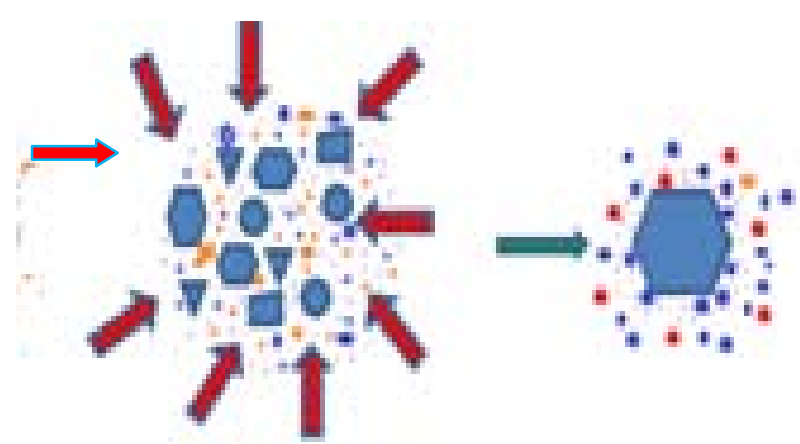

Forces in Nature (Gravitational,Nuclear,

Electomagnetics etc.)

Convergence of Particles

Figure. 1.2: Big- Crunch Phase

\section{B. Satellite Image Classification}

Satellite images are important and powerful tool to get information of land cover features of inaccessible remote areas. They provide qualitative and quantitative information and also reduce field work and time. These images have variety of uses, including: cartography, military intelligence and meteorology. Satellite image classification involves identification of different land cover classes on the image. Satellite image classification plays an important role in extracting and interpretation of important information from satellite images which could be required for variety purposes like - Spatial data mining [14], Extract information for an application, Thematic map creation, Field surveys, Effective decision making, Disaster management, studying various vegetation types like agriculture or forest, various land uses of urban area etc.

Rest of the paper is structured as follows - Section 2 depicts BBBC optimization algorithm. Section 3 contains the proposed work. Section 4 contains Result and Discussions and Section 5 contains Future work and References.

\section{BB-BC OPTIMIZATION Algorithm}

Big Bang-Big Crunch (BB-BC) optimization algorithm is used to find optimal solution of an objective function $\mathrm{f}(\mathrm{x})$.The algorithm has been divided into two phases (i) Big Bang (ii) Big Crunch.

In the Big-Bang phase particles are spread randomly in the space and in the Big-Crunch Phase randomly distributed particles are concentrated into one place known as center of mass which (CM) which is calculated according to the following equation:

$$
C M=\frac{\sum_{i=0}^{i=N} \frac{x_{i}}{f\left(x_{i}\right)}}{\sum_{i=0}^{i=N} \frac{1}{f\left(x_{i}\right)}}
$$

Where $x_{i}$ is the $i^{\text {th }}$ particle in the D-Dimensional search space, $f\left(x_{i}\right)$ is the fitness value of the point $x_{i}$ for the objective function $\mathrm{f}(\mathrm{x}), \mathrm{N}$ is the population size in the Big Bang Phase.

After the Big Crunch phase, Big Bang Phase follows in which the candidate solutions are again spread in the search space within the limits of the search space around the center of mass of the last phase for better convergence using the formula:

$$
\mathrm{CM}=\mathrm{CM}+\mathrm{lr} / \mathrm{j}
$$

Where $l$ is the limit of the search space,

$r$ is the random number in $[0,1]$

$\mathrm{j}$ is the iteration step

The described Big Bang - Big Crunch phase is continued till a termination criterion is met. The same is illustrated by the figure. 2
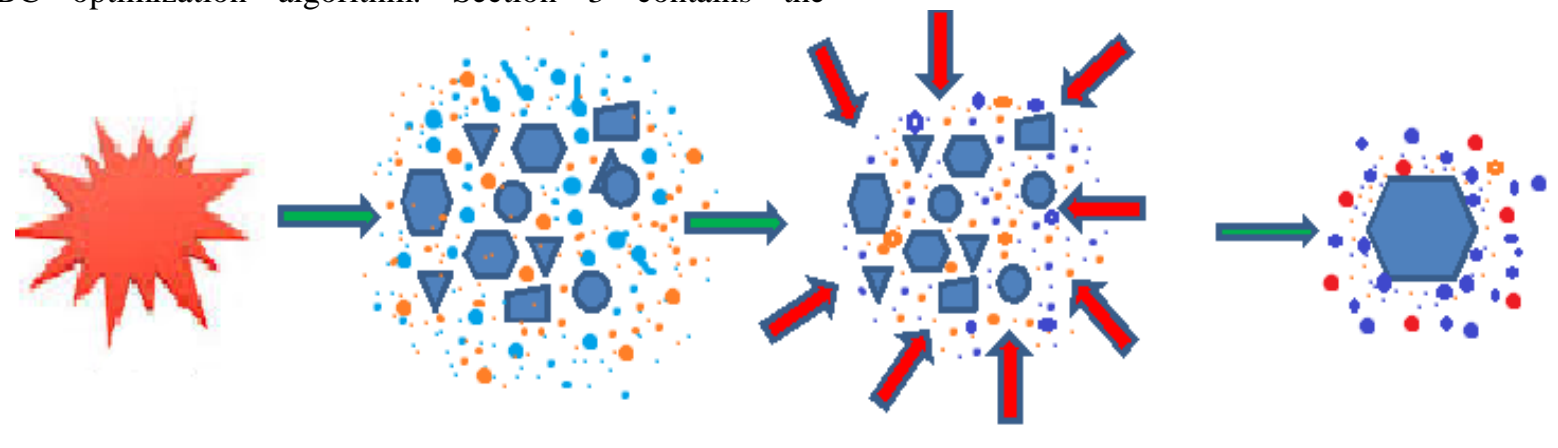

Forces in nature (Gravitational, Nuclear, Electromagnetic etc.)

\section{Explosion in nature}

$$
\text { Random Spread }
$$

$$
\text { Convergence of particles }
$$

$$
\mathbf{C M}=\mathbf{C M}+\mathbf{l r} / \mathbf{j}
$$

\section{Big Bang Phase}

Figure 2: Big Bang-Big Cruch Optimization Algorithm

\section{Big Crunch Phase}

III.

\section{A. Pseudo Code of BBBC}

Assumptions:
Objective Function $f(x)$ : The function to be optimized. Termination Criteria: Number of Iterations should be less than a fixed number. 
Fitness $\mathrm{f}\left(\mathrm{x}_{\mathrm{i}}\right)$ : Value of Objective Function $\mathrm{f}(\mathrm{x})$ at a point $\mathrm{x}_{\mathrm{i}}$.

$>$ Initialize the particles randomly in search space.

$>$ While the termination criteria is not met

- Compute the center of mass of particles using eq.(1) (Big-Crunch Phase)

- Compute the fitness of the center of mass.

- Spread new particles around center of mass using eq.(2) (Big Bang Phase)

\section{$>$ End While}

$>$ Output $\mathrm{CM}$ as the solution of the objective function $f(x)$

$>$ End

\section{PROPOSED WORK}

\section{A. Dataset Used}

For the purpose of feature extraction a multi-spectral, multi resolution and multi-sensor 7-band satellite image of Alwar City (Rajasthan, India) of size 472 X 546 has been used.

These bands are Red, Green, Near Infra Red (NIR), Middle Infra Red (MIR), Radarsat -I (RS1), radarsat -2 (RS2) and Digiatl elevation Model (DEM) as shown in figure. 3(a-g).

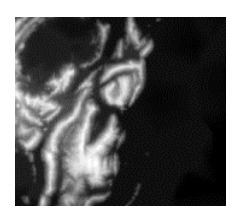

Figure 3(a) Dem

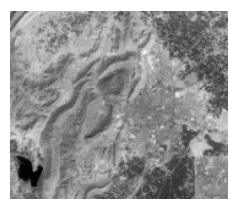

Figure 3(d) NIR

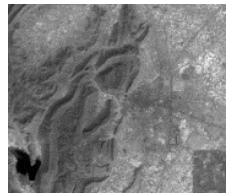

Figure 3(b) Green

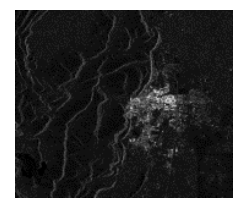

Figure 3(e) RS1

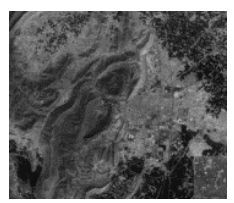

Figure 3(g) Red

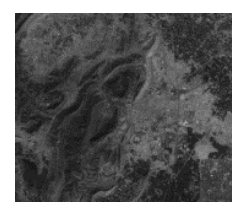

Figure 3(c) MIR

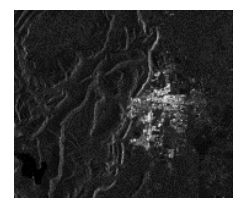

Figure 3(f) RS2
The sample training data of each class as produced by the expert is shown in Figure.4. $2 / 3^{\text {rd }}$ of the training data has been used for development of the classifier based on BBBC optimization algorithm and $1 / 3^{\text {rd }}$ is used as validation/reference data.

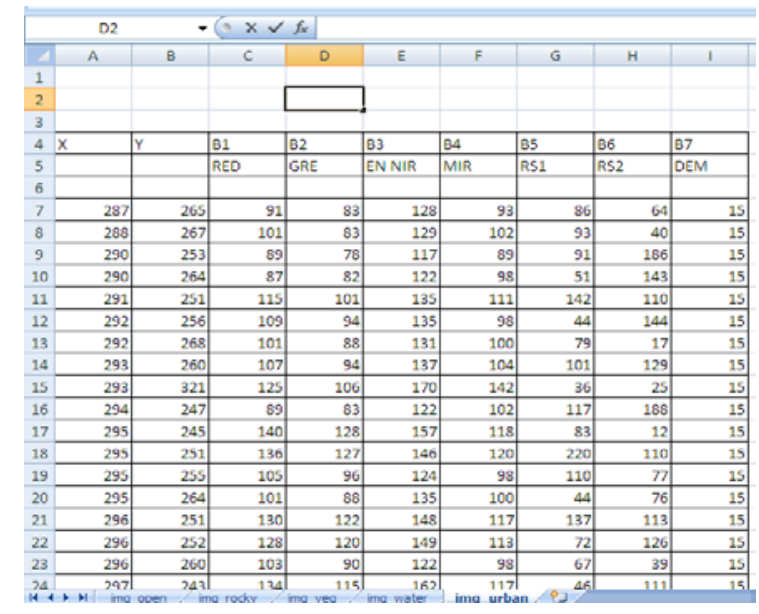

Figure 4: Sample data for the classes produced by the expert.

\section{B. Proposed Methodology}

To classify a pixel in the satellite image known as Test Particle (TP) we have calculated the fitness of that pixel (TP) in all the classes in which it is to be classified. To calculate the fitness we have taken the objective function as the Euclidian distance of digital number of TP in each of the 7 bands from the digital number of Center of mass

For that we have used the proposed modified Big BangBig Crunch algorithm by using which we first have taken any 20 pixels of the training data of each class produced by the expert and calculated the Euclidian distance of each of those pixels in all the bands from the test particle (TP) and calculated the center of mass (CM). Next we calculated the fitness of the CM and compare with all the 20 particles and if any particle has less fitness than CM we made that particle $\mathrm{CM}$. Then we again went to Big Bang phase and spread the particle around CM but within the limits of the search space using random number generators and calculate the fitness of the new particles and produced new center of mass. The process is repeated for certain number of times, 40 in our case as after 20-30 iterations we found that the fitness of the CM can't be further improved as shown by the graph in figure 5 . After calculating the fitness of TP with each of the class we compare them to find the class in which the fitness is best and $\mathrm{TP}$ is assumed to belong to that class.

\section{Proposed Algorithm}

\section{Algorithms: Big Bang -Big Crunch based satellite image classification}

1. Get the multispectral satellite image.

2. Take the classes of the land cover (produced by the expert) Water, Urban, Rocky, Barren and Vegetation having training pixels. Take the upper \& lower bound of each class.

3. Take first $\mathrm{N}$ ( $\mathrm{N}=20$ in our case) training pixels of each class and consider them as first $\mathrm{N}$ particles in the universe.

4. Take any pixel from the image as a Test-Particle (TP) to be classified in any one of the classes given by the expert.

5. Repeat the following 40 times steps for each class - Water, Urban, Rocky, Barren and Vegetation to find fitness f1, f2, f3, f4\& f5 of the test particle (TP) for each of the class respectively.

a) Calculate the fitness of each of the $\mathrm{N}$ training pixel/candidate solution based upon the Euclidean Distance of the pixel value of each band from the TP. 
b) Calculate the center of mass using the formula:

$$
C M=\frac{\sum_{i=0}^{i=N} \frac{x_{i}}{f\left(x_{i}\right)}}{\sum_{i=0}^{i=N} \frac{1}{f\left(x_{i}\right)}}
$$

c) Calculate the fitness of the center of mass (cmf)

d) Compare the fitness of the center of mass (cmf) with each of the $\mathrm{N}$ candidate solutions. If any particle has less fitness as center of mass, make that particle center of mass.

e) Spread the particles again around the center of mass within the bounds to get new $\mathrm{N}$ candidate solutions using formula

$$
\mathrm{CM}+\mathrm{l} * \mathrm{r} / \mathrm{j}
$$

Where $l$ is the bounds, $r$ is random number and $j$ is the iteration step.

6. Compare each of the fitness f1, f2, f3, f4 \& f5. Absorb the test particle in the class having least fitness.

7. Take another point from the image as TP and go to step 5 until all the particles are classified.

8. END

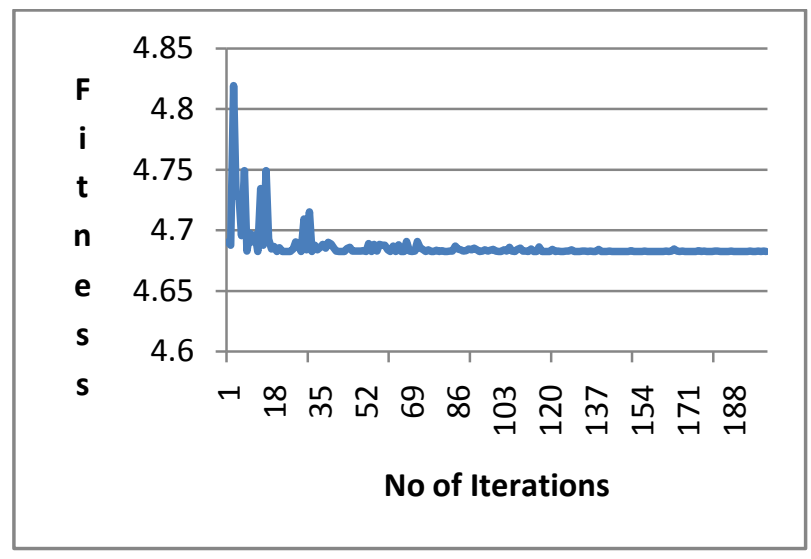

Figure 5: Variation of Fitness with No. of Iterations

\section{Results AND Discussions}

After applying the proposed algorithm on the 7-band of Alwar image the classified image is obtained with different classes like vegetation, water, rocky, urban and barren. The different colors define the different terrain features in this image. The Green color represents vegetation region, Blue color represents water region, Red color represents rocky region, Yellow color represents urban region \& Magenta color represents barren region respectively. This classified image can be compared with the original satellite image as shown in figure 6 .

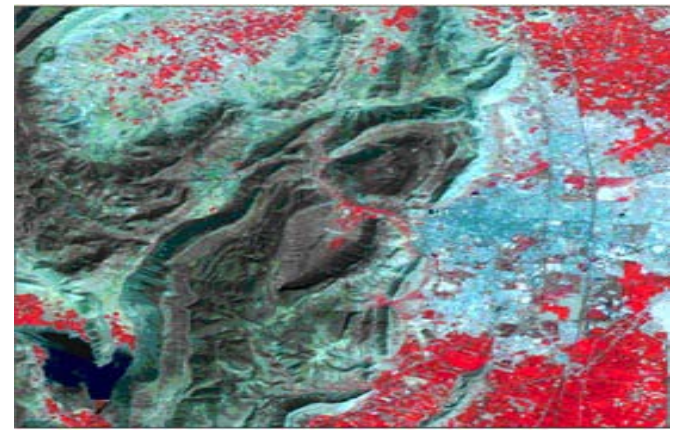

Figure 6(a-b): Comparison of (a) Original Alwar image and

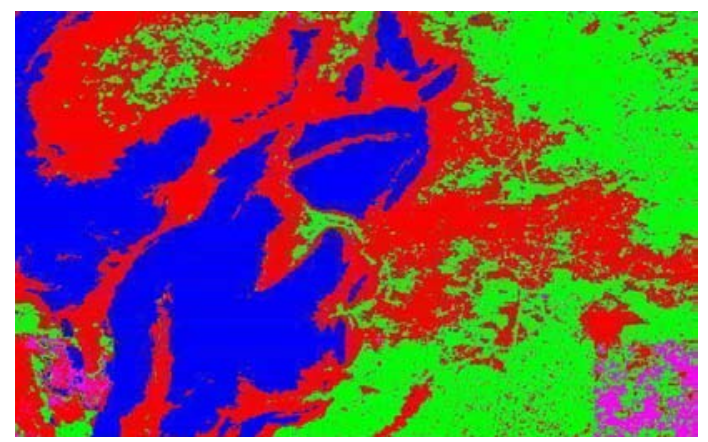

Figure 6(b): Classified image.

\section{A. Accuracy Assessment}

To determine the accuracy of the proposed algorithm we have to find accuracy in the image classification. The aim is to quantitatively determine how effectively the pixels were grouped into correct feature classes in the area under investigation. Classification accuracy of our proposed algorithm is expressed using classification error matrix [2]. Error matrices compare, on a category by category basis, the relationship between known reference data/validation data (ground truth) and the corresponding results of an automated classification. A set of randomly selected reference pixels is used for experimentation. Reference pixels are points on the classified image for which actual features are known. For the purpose of validation we have taken 150 vegetation pixels, 190 Urban pixels, 200 Rocky pixels, 70 water pixels and 80 barren land pixels from the training set and the error matrix obtained is shown below. The error matrix interpretation along column suggests how many pixels are classified correctly by the algorithm. For e.g. in the forth column, out of 190 Urban pixels, 181 pixels were classified correctly into Urban by the proposed algorithm, 6 were misclassified as Barren, 2 as vegetation and 1 as rocky.

Table 1: Error Matrix of BBBC

\begin{tabular}{|c|c|c|c|c|c|c|}
\hline Feature & Rocky & Water & Barren & Urban & Vegetation & Total \\
\hline Rocky & 127 & 0 & 8 & 1 & 0 & 136 \\
\hline Water & 70 & 70 & 0 & 0 & 0 & 140 \\
\hline Barren & 3 & 0 & 66 & 6 & 0 & 75 \\
\hline Urban & 0 & 0 & 6 & 181 & 0 & 187 \\
\hline Vegetation & 0 & 0 & 0 & 2 & 150 & 152 \\
\hline Total & 200 & 70 & 80 & 190 & 150 & 690 \\
\hline
\end{tabular}


Various factors that can be considered for the accuracy assessments are explained with their calculated values as follows:

Kappa Coefficient: The kappa coefficient is used to interpret the results of error matrix. It is a discrete multivariate technique. The Kappa statistics incorporates the observations on the diagonal as well as the off diagonal which gives more robust assessment of accuracy than other accuracy measures. The Kappa coefficient of the Alwar image can be calculated by applying following formula to the Error Matrix:

$$
\mathrm{K}=\frac{\mathrm{N} \sum_{i=1}^{r} x_{i i}-\sum_{i=1}^{r}\left(x_{i+\cdot} \cdot x_{+i}\right)}{N^{2}-\sum_{i=1}^{r}\left(x_{i+} \cdot x_{+i}\right)}
$$

$\mathrm{r}=$ Number of rows in the error matrix $(\mathrm{r}=5$ in our case)

$x_{i i}=$ the number of observations in row $i$ and column $i$ ( on the major diagonal)

$\mathrm{x}_{\mathrm{i}^{+}}=$the total number of observations in row $\mathrm{i}$ (shown as marginal total to right of the matrix)

$\mathrm{x}_{+\mathrm{i}}=$ the total number of observations in column $\mathrm{i}$ (shown as marginal total at the bottom of the matrix)

$\mathrm{N}=$ Total numbers of observations included in the matrix $(\mathrm{N}=$ 690 in our case)

The Kappa (K) coefficient of the Alwar image is 0.8233 which indicates that an observed classification is $82.33 \%$ better than one resulting from chance.

Producer's Accuracy: Producer's accuracy measures that how much of the land in each class was classified correctly. In other words it indicates that how much the image is classified correctly category wise by the analyst. It is calculated as:

$$
\alpha=\frac{\begin{array}{c}
\text { No. of correctly classified pixels } \\
\text { in each category (on major diagonal) }
\end{array}}{\begin{array}{c}
\text { Total No. of training set pixels } \\
\text { used for that category (the column total) }
\end{array}}
$$

Producer's accuracy of the proposed BBBC based classifier is shown in Table 2.

User's accuracy: User's accuracy measures how well the classification performed in the field by category (rows). It is calculated as:

$$
\begin{aligned}
& \text { No. of correctly classified pixels } \\
& \beta=\frac{\text { in each category (on major diagonal) }}{\text { Total No. of pixels used }}
\end{aligned}
$$

User's accuracy of the proposed BBBC based classifier is shown in Table 2.

Table 2: Different Accuracy Measures For Alwar Image

\begin{tabular}{|c|c|c|c|c|c|}
\hline \multirow{2}{*}{ Accuracy } & \multicolumn{5}{|c|}{ Features (\%) } \\
\cline { 2 - 6 } & Vegetation & Urban & Rocky & Water & Open \\
\hline $\begin{array}{c}\text { Producer's } \\
\text { Accuracy }\end{array}$ & 100 & 96.79 & 93.38 & 100 & 88 \\
\hline $\begin{array}{c}\text { User's } \\
\text { Accuracy }\end{array}$ & 100 & 95.26 & 63.5 & 100 & 82.5 \\
\hline
\end{tabular}

Based upon the Producer's Accuracy and User's Accuracy from Table 2 we find that Big Bang- Big Crunch based classifier gives $100 \%$ result for Vegetation \& water and 95.26\% result for Urban area as depicted by Figure. 7. So we can say that the algorithm has tendency towards vegetation, water and urban area.

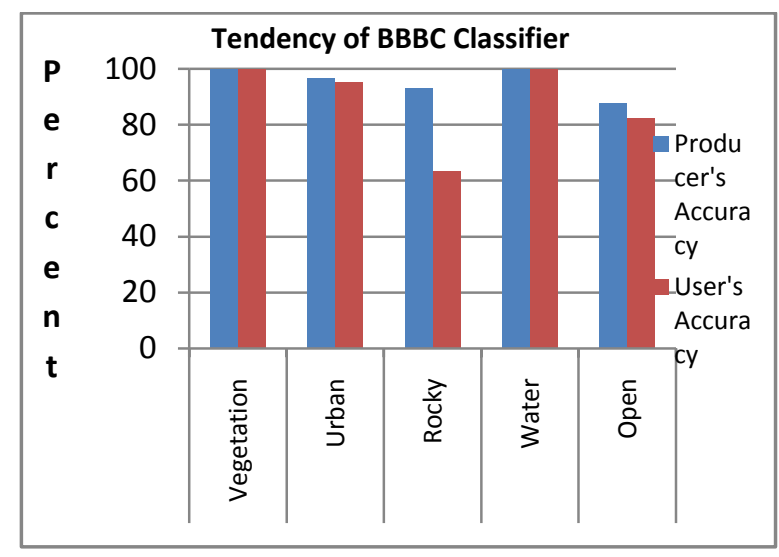

Figure 7: Tendency of BBBC classifier for different Terrain Features

Overall Accuracy: Overall accuracy is measure of the number of correct observations divided by the total number of classifications. This is very crude measure of accuracy and is calculated as below:

$$
\gamma=\frac{\begin{array}{c}
\text { No. of correct classifications } \\
\text { (Sum of all values on major diagonal) }
\end{array}}{\text { Total No. of classifications }}
$$

The overall accuracy of the proposed BBBC based classifier is 0.86 .

\section{B. Comparison with other nature inspired algorithms}

The results are compared with other nature inspired algorithms like Biogeography based Optimization (BBO) [18], Particle Swarm Optimization (PSO) [4], Hybrid Rough Set \& Biogeography Based optimization [19], Hybrid Biogeography Based optimization \& Flower Pollination by Artificial bees [12], Hybrid Fuzzy System \& Biogeography Based optimization [19], Hybrid Ant Colony optimization \& Self Organising Feature Map [13] and Hybrid Ant Colony Optimization \& Biogeography Based optimization [19]. For this we have taken the three parameters:

1) Error Matrices: We have taken the Error matrices for different nature inspired algorithms for comparison purposes which is represented by Table 3 -9.

Table 3: Error Matrix of BBO [18]

\begin{tabular}{|c|c|c|c|c|c|c|}
\hline Feature & Rocky & Water & Barren & Urban & $\begin{array}{c}\text { Vegetatio } \\
\mathrm{n}\end{array}$ & Total \\
\hline Rocky & 175 & 0 & 3 & 15 & 0 & 194 \\
\hline Water & 0 & 70 & 0 & 0 & 0 & 70 \\
\hline Barren & 26 & 0 & 128 & 81 & 21 & 256 \\
\hline Urban & 0 & 0 & 39 & 92 & 1 & 129 \\
\hline $\begin{array}{c}\text { Vegetati } \\
\text { on }\end{array}$ & 1 & 0 & 0 & 1 & 127 & 129 \\
\hline \begin{tabular}{c} 
Total \\
\hline
\end{tabular} & 202 & 70 & 170 & 189 & 150 & 781 \\
\hline
\end{tabular}


Sunita Sharma et al, International Journal of Advanced Research in Computer Science, 8 (8), Sept-Oct 2017,186-192

Table 4: Error Matrix attained of PSO [4]

\begin{tabular}{|c|c|c|c|c|c|c|}
\hline Feature & Rocky & Water & Barren & Urban & $\begin{array}{c}\text { Vegetati } \\
\text { on }\end{array}$ & Total \\
\hline Rocky & 286 & 0 & 0 & 3 & 18 & 307 \\
\hline Water & 0 & 59 & 0 & 0 & 28 & 87 \\
\hline Barren & 0 & 0 & 24 & 7 & 1 & 32 \\
\hline Urban & 0 & 0 & 7 & 22 & 4 & 33 \\
\hline $\begin{array}{c}\text { Vegetatio } \\
\text { n }\end{array}$ & 0 & 47 & 0 & 0 & 79 & 126 \\
\hline \begin{tabular}{c} 
Total \\
\hline
\end{tabular} & 286 & 106 & 31 & 32 & 130 & 585 \\
\hline
\end{tabular}

Table 5: Error Matrix of Hybrid Rough/BBO [19]

\begin{tabular}{|c|c|c|c|c|c|c|}
\hline Feature & Rocky & Water & Barren & Urban & Vegetation & Total \\
\hline Rocky & 176 & 1 & 17 & 2 & 6 & 202 \\
\hline Water & 3 & 69 & 0 & 0 & 0 & 72 \\
\hline Barren & 20 & 0 & 119 & 91 & 17 & 247 \\
\hline Urban & 1 & 0 & 32 & 88 & 0 & 121 \\
\hline Vegetation & 0 & 0 & 2 & 9 & 127 & 138 \\
\hline Total & 200 & 70 & 170 & 190 & 150 & 780 \\
\hline
\end{tabular}

Table 6: Error Matrix of Hybrid FPAB/BBO [12]

\begin{tabular}{|c|c|c|c|c|c|c|}
\hline Feature & Rocky & Water & Barren & Urban & Vegetation & Total \\
\hline Rocky & 181 & 0 & 28 & 0 & 10 & 219 \\
\hline Water & 0 & 70 & 0 & 0 & 0 & 70 \\
\hline Barren & 17 & 0 & 105 & 89 & 2 & 213 \\
\hline Urban & 2 & 0 & 36 & 90 & 0 & 128 \\
\hline Vegetation & 0 & 0 & 1 & 11 & 138 & 150 \\
\hline Total & 200 & 70 & 170 & 190 & 150 & 780 \\
\hline
\end{tabular}

Table 7: Error Matrix of Hybrid Fuzzy/BBO [19]

\begin{tabular}{|c|c|c|c|c|c|c|}
\hline Feature & Rocky & Water & Barren & Urban & $\begin{array}{c}\text { Vegetat } \\
\text { ion }\end{array}$ & Total \\
\hline Rocky & 173 & 0 & 3 & 15 & 0 & 192 \\
\hline Water & 0 & 70 & 0 & 0 & 0 & 70 \\
\hline Barren & 26 & 0 & 128 & 81 & 21 & 255 \\
\hline Urban & 0 & 0 & 39 & 93 & 1 & 130 \\
\hline Vegetation & 1 & 0 & 0 & 1 & 127 & 129 \\
\hline Total & 200 & 70 & 170 & 190 & 150 & 780 \\
\hline
\end{tabular}

Table 8: Error Matrix of Hybrid ACO/SOFM [13]

\begin{tabular}{|c|c|c|c|c|c|c|}
\hline Feature & Rocky & Water & Barren & Urban & $\begin{array}{c}\text { Vegeta } \\
\text { tion }\end{array}$ & Total \\
\hline Rocky & 161 & 0 & 5 & 0 & 6 & 172 \\
\hline Water & 2 & 65 & 1 & 1 & 0 & 69 \\
\hline Barren & 11 & 0 & 110 & 46 & 0 & 167 \\
\hline Urban & 21 & 0 & 47 & 121 & 0 & 189 \\
\hline Vegetation & 5 & 5 & 7 & 22 & 144 & 183 \\
\hline Total & 200 & 70 & 170 & 190 & 150 & 780 \\
\hline
\end{tabular}

Table 9: Error Matrix of Hybrid ACO/BBO [19]

\begin{tabular}{|c|c|c|c|c|c|c|}
\hline Feature & $\begin{array}{c}\text { Rock } \\
\mathrm{y}\end{array}$ & Water & Barren & Urban & $\begin{array}{c}\text { Vegetatio } \\
\mathrm{n}\end{array}$ & Total \\
\hline Rocky & 168 & 0 & 10 & 2 & 2 & 182 \\
\hline Water & 1 & 70 & 1 & 0 & 3 & 75 \\
\hline Barren & 27 & 0 & 125 & 27 & 0 & 179 \\
\hline Urban & 0 & 0 & 26 & 127 & 0 & 153 \\
\hline $\begin{array}{c}\text { Vegetati } \\
\text { on }\end{array}$ & 4 & 0 & 8 & 34 & 145 & 191 \\
\hline \begin{tabular}{c} 
Total \\
\hline
\end{tabular} & 200 & 70 & 170 & 190 & 150 & 780 \\
\hline
\end{tabular}

Comparison of different terrain features of different nature inspired algorithms based upon the error matrices is represented by the figure 8 .

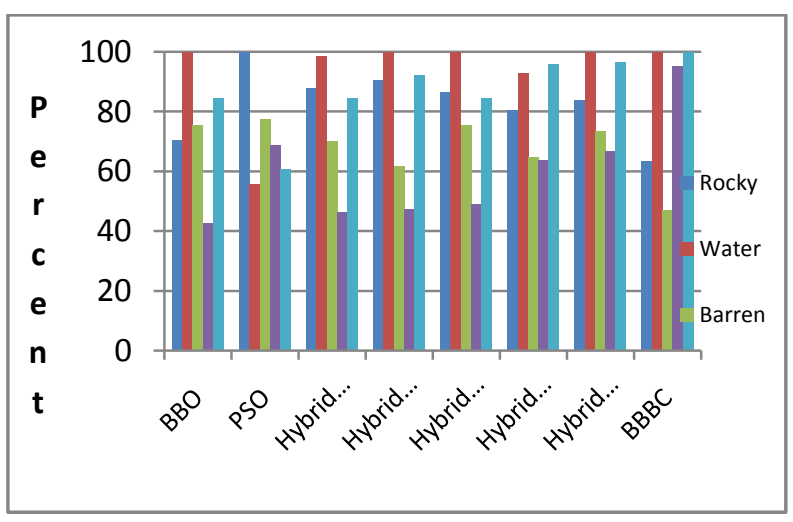

Figure 8: Comparison of Different Terrain Features Identification by different nature inspired algorithms

This indicates that BBBC optimization gives better performance for Rocky, Urban, Vegetation and water.

2) Kappa Coefficient: The value of the Kappa coefficient for the proposed algorithm is 0.8233 which shows that the proposed classification is better as the Kappa coefficient of some other algorithms are as shown in Fig.9.

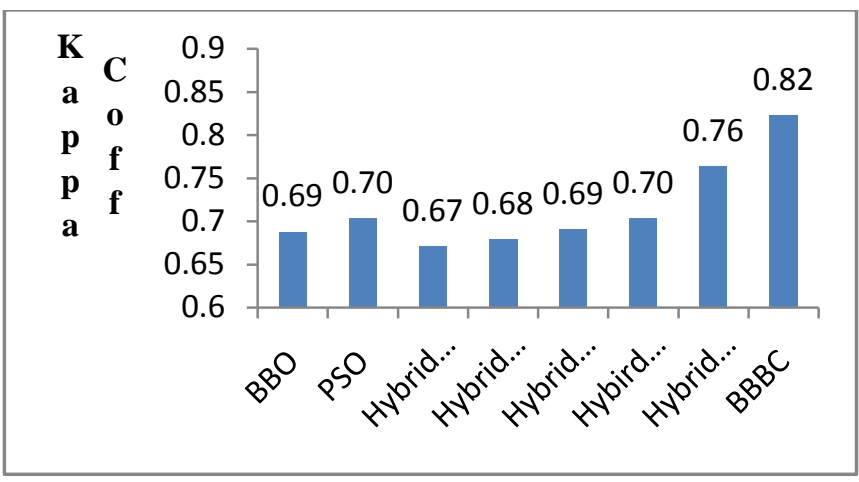

Figure 9: Comparison of Kappa Coefficient of Different Nature Inspired Algorithms

Kappa coefficient of Biogeography based Optimization (BBO) [18] is 0.69, Particle Swarm Optimization (PSO) [4] is 0.70, Hybrid Rough Set \& Biogeography Based optimization [19] is 0.67, Hybrid Biogeography Based optimization \& Flower Pollination by Artificial bees [12] is 0.68, Hybrid Fuzzy System \& Biogeography Based optimization [19] is 0.69, Hybrid Ant Colony optimization \& Self Organising Feature Map [13] is 0.70 and Hybrid Ant Colony Optimization 
\& Biogeography Based optimization [19] is 0.76 as illustrated by Fig 9.

3) Overall Accuracy: The overall accuracy for satellite image classification for the proposed algorism is 0.86 which shows that the observed classification is better as the value of overall accuracy of BBO, PSO, Hybrid Rough/BBO, Hybrid FPBAB/BBO, Hybrid Fuzzy/BBO, Hybrid ACO/SOFM, Hybrid ACO/BBO are 0.76, 0.80, 0.74, 0.75,0.76, 0.77, 0.81, respectively as illustrated by Figure 10 .

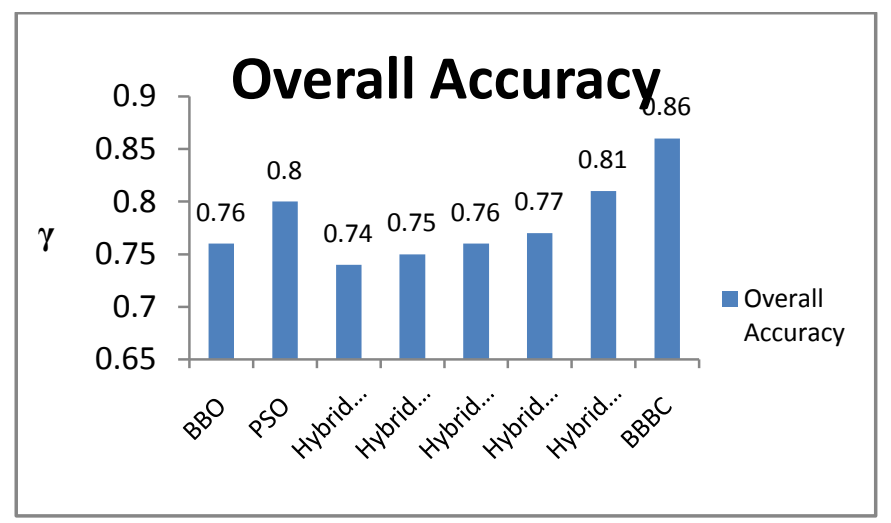

Figure 10: Comparison of Overall Accuracy of different nature inspired algorithms

\section{CONCLUSION AND FUTURE WORK}

In this paper we have used Big Bang- Big Crunch algorithm as an efficient land cover classifier for satellite image. The results produced by the algorithm are highly significant which indicates that the BBBC is an efficient classification tool for satellite image

The future scope of the algorithm is that it can be hybrid with other nature inspired technique or implemented with different random number generator functions like Lèvy distribution for spreading the particle in the space, so that Kappa coefficient can be further improved. Also the results suggest that BBBC algorithm proved to be an efficient algorithm and can also be applied effectively in other areas of research like medical imaging etc

\section{REFERENCES}

[1] Osman K. Erol, Ibrahim Eksin. "A new optimization method : Big Bang - Big Crunch”. Advances in Engineering Software. 37 (2006) 106-111

[2] Lillesand T. M. Keifer, R. W., Chipman, J. W. (2004), "Remote Sensing and Interpretation", New York: John Wiley \& Sons Ltd. pp .586 -592.

[3] Panchal V. K., Singh P. Kaur N., Kundra H. "Biogeography based Satellite Image Classification”, International Journal of
Computer Science and Information Security, Vol. 6, No 2, 2009. Mumbai, India pp:165-170.

[4] Panchal V. K., Singh P. Kaur N., Kundra H. "Comparative study of Particle Swarm Optimization based unsupervised clustering techniques" , International Journal of Computer Science and Information Security, Vol. 9, 132-140, 2009.

[5] Goel L., Gupta D., Panchal V.K. "Performance governing factors of Biogeography based land cover feature extraction: An analytical study". Proceedings of the World Congress on Information and communication Technologies Dec 11-14, 2011,

[6] Sakthivel S., Pandiyan S.A., Marikani S., Selvi S.K.., "Application Of Big Bang- Big Crunch Algorithm for Optimal Power Flow Problems", The International Journal of Engineering And Science, Vol 2,Issue 4 pg. 41-47 (2013).

[7] Labbi Y., Attous D. B. ,’Big Bang -Big Crunch Optimization Algorithm for Economic Disptach with Valve-Point Effect”, Journal of Theoretical and Applied Information Technology (2010).

[8] Kumar S, Bhalla P Singh A, "Fuzzy Rulebase Generation from Numerical Data using Big Bang - Big Crunch optimization”, IE(I) Journal - ET Vol. 91 (2011)

[9] Jordehi A. R.," A chaotic- based big bang- big crunch algorithm for solving global optimization problems", Neural computing and application 25:1329-1335 (2014)

[10] Naim S., Hagras H., “A Big Bang- Big Crunch optimized general Type-2 Fuzzy Logic approach for multi-criteria group decision making”, JAISCR, Vol. 3, No. 2, PG. 117-132 (2013)

[11] Tabrizian Z., Afshan E., Amiri G.G., Beigy M.H.A., Nejaz S.M.P., A new damage detection method : Big Bang- Big Crunch (BB-BC) algorithm (Shock and vibration 20, 633-648)

[12] Johal, N. K., Singh, S., \& Kundra, H. (2010). A hybrid FPAB/BBO algorithm for satellite image classification. International Journal of Computer Applications (0975-8887), 6(5).

[13] Goel, S., Sharma, A., \& Panchal, V. K. (2011). A Hybrid Algorithm for Satellite Image Classification. In Advances in Computing, Communication and Control (pp. 328-334). Springer Berlin Heidelberg.

[14] Soliman, O, S. and Mahmoud, A.S., 2012. "A classification system for remote sensing satellite images using support vector machine with non-linear kernel functions”, In proc. 8th International Conference on Informatics and Systems (INFOS), IEEE, 14-16 May 2012, pp.BIO-181,BIO-187, Cairo.

[15] Ooseph Silk. "Horizons of Cosmology". Templeton Press. p. 208. (2009)

[16] Simon Singh. "Big Bang: The Origin of the Universe”. Harper Perennial. p. 560. (2005)

[17] Wollack, E. J. 2010"Cosmology: The Study of the Universe".Universe 101: Big Bang Theory. NASA. Archived from the original on 14 May 2011. Retrieved 27 April 2011.

[18] Panchal, V. K., Goel, S., \& Bhatnagar, M. (2009, December). Biogeography based land cover feature extraction. In Nature \& Biologically Inspired Computing, 2009. NaBIC 2009. World Congress on (pp. 1588-1591). IEEE.

[19] Goel, S., Sharma, A., Bedi, P., \& Panchal, V. K. (2011). Decision Gap within Swarm in Natural Terrain Feature Identification. International Journal of Computer Science and Security (IJCSS), 1(3), 1. 